\title{
Robótica Educacional e Computação Desplugada: Experiência em Oficinas para Calouros
}

\author{
Breno Sousa ${ }^{1}$, Dener Ripardo ${ }^{1}$, Izabela Campos ${ }^{1}$, Jacqueline Maciel $^{1}$, \\ Rafael Santos $^{1}$, Waldinei Romano ${ }^{1}$, Alberto Alan Raiol, Fábio Bezerra ${ }^{1}$ \\ ${ }^{1}$ Instituto Ciberespacial - Universidade Federal Rural da Amazônia (UFRA) \\ Avenida Presidente Tancredo Neves, 2501 - Belém-Pará \\ fabio.bezerradufra.edu.br
}

\begin{abstract}
Information technology is an area rich in job opportunities and innovation and therefore attracts many students. Nevertheless, the Educational Institutions (EI) that are home to courses in this area also has problems with the retention of students, a fact that has impact on the financial health of them. This paper describes how the experience with freshmen can help mitigate this problem. The results reported here are useful for stimulating similar actions in other EI. In this case, Educational Robotics and Unppluged Computing actually functioned as a language of welcome and stimulation in the field, not, as one might suppose, a childish language.
\end{abstract}

Resumo. A informática é uma área rica em oportunidades de trabalho e inovação e por isso atrai muitos estudantes. Apesar disso, as Instituições de Ensino Superior (IES) que abrigam cursos nessa área também têm problemas com a retenção de alunos, fato que tem impacto na saúde financeira dessas IES. Ações que mitiguem esse problema são importantes para as IES e para a sustentabilidade da indústria que demanda esses profissionais. Este trabalho descreve como a experiência com a Semana do Calouro pode mitigar esse problema. Os resultados relatados aqui são úteis para estimular ações semelhantes em outras IES. A Robótica Educacional e a Computação Desplugada funcionaram como uma linguagem de acolhimento e estímulo a área, e não, como poderia se supor, uma linguagem infantil ou que ridicularizasse a maturidade dos calouros.

\section{Introdução}

A Universidade Federal Rural da Amazônia (UFRA) está localizada no estado do Pará, com vários campi no interior do estado e na capital Belém, que é seu campus sede. Por muitos anos foi dedicada à formação de profissionais e a pesquisa nas áreas de agrárias (Agronomia, Engenharia Florestal, Veterinária e Zootecnia). Em 2009, a UFRA criou dois cursos na área de informática, com a oferta anual de 50 vagas em cada um deles, sendo eles: Licenciatura em Computação (LC) e Bacharelado em Sistemas de Informação (BSI). Esses cursos têm como objetivo atender uma demanda regional de profissionais de informática, mas também estimular a inovação na área de Tecnologia da Informação e Comunicação (TIC) aplicada às áreas agrárias.

Apesar das evidentes oportunidades que a área de TIC tem com a área de agrárias, além das diferentes demandas de TIC no estado, os cursos de LC e BSI da UFRA não estão imunes aos problemas relacionados com a retenção (tempo de conclusão 
do curso superior ao do projeto pedagógico) de alunos. Especificamente, nesses cursos de informática, os graduandos relatam dificuldades em disciplinas de algoritmos e programação de computadores, afirmação que pode ser confirmada também com o elevado índice de reprovação e desempenho médio em avaliações de turmas anteriores. Essas disciplinas exigem dos alunos autonomia, criatividade e habilidades como interpretação de problemas, desenvolvimento lógico, pensamento computacional, além do domínio de conceitos matemáticos básicos provenientes do ensino médio.

Esses inúmeros requisitos podem ser um dos fatores que contribuem para essa evidente dificuldade dos alunos dos cursos de informática nessas disciplinas. Assim, a experiência na UFRA e diversos relatos em outras universidades, como os apresentados em [Raiol et al. 2015, Dantas et al. 2015, Ribas et al. 2016] e discutidos na Seção 2, são evidências que nos convidam a propor soluções que mitiguem os problemas com a retenção de alunos na área de informática.

Uma das estratégias adotadas pela UFRA é destinar uma semana para acolhida dos seus alunos calouros, que normalmente é organizada pelos centros acadêmicos de cada curso, com o apoio institucional das coordenadorias e pró-reitorias. Trata-se da Semana do Calouro, evento que, guardando-se as diferenças locais de cada curso e região do Brasil, é repetido em formato semelhante em todo o país, e tem objetivos de socializar os calouros com os veteranos, apresentar a administração e rotina da Universidade, e apresentar o curso no qual o aluno foi admitido.

No caso da Semana do Calouro 2018 da UFRA, campus Belém, dois dias foram reservados a oferta de seis oficinas de formação tecnológica, das quais duas são objeto de avaliação deste artigo: Computação Desplugada e Robótica Educacional. Além das oficinas de formação tecnológica, a semana contou com um dia dedicado a apresentação das unidades gestoras e outro dia para socialização dos calouros (ver Seção 3).

Este trabalho descreve uma experiência de acolhida dos calouros, mas também discute como intervenções semelhantes podem mitigar o problema da retenção de alunos, especialmente os ligados a área de informática. Os resultados apresentados na Seção 4 são evidências para que experiências semelhantes sejam repetidas nos próximos anos, tanto na UFRA como em IES que ofertam cursos de informática. Na Seção 5, é relatado uma síntese da experiência e oportunidades para trabalhos futuros.

\section{Trabalhos Relacionados}

O núcleo dos cursos de informática exige dos alunos habilidades e competências como a criação e implementação de algoritmos e programas de computador. As dificuldades apresentadas pelos alunos nas disciplinas iniciais de algoritmo e programação de computadores podem aumentar os índices de retenção desses alunos no curso. Na Seção 2.1 apresentamos alguns trabalhos relacionados aos esforços da comunidade para entender e mitigar os problemas com a retenção e evasão de alunos.

Considerando que este trabalho avalia a intervenção de duas oficinas nesse processo de mitigação da retenção, apresentamos na Seção 2.2 alguns trabalhos sobre a adoção da Robótica Educacional, enquanto que na Seção 2.3 é exposto alguns trabalhos sobre a adoção da Computação Desplugada, apontando o que a comunidade tem feito para adotar esses conteúdos na educação básica e superior. 
VII Congresso Brasileiro de Informática na Educação (CBIE 2018)

Anais dos Workshops do VII Congresso Brasileiro de Informática na Educação (WCBIE 2018)

\subsection{Retenção de Alunos}

Vários fatores podem contribuir com retenção (ou evasão) de alunos em um curso de graduação, mas para graduandos da área de TIC, uma das principais causas está relacionada ao desempenho em disciplinas que envolvem programação de computadores, como pode ser observado nos trabalhos apresentados em [de Macedo and Prietch 2013, Franz et al. 2014, Dantas et al. 2015, Raiol et al. 2015, Ribas et al. 2016].

Há esforços de mitigação do problema que consideram uma abordagem de ensino mais técnica ou profissionalizante[de Macedo and Prietch 2013, Franz et al. 2014]. No caso, em [de Macedo and Prietch 2013] os autores relatam a experiência com o kit de microcontroladores, placas e sensores Arduino, com alunos do curso de Licenciatura em Informática e Sistemas de Informação da Universidade Federal de Mato Grosso (UFMT), já em [Franz et al. 2014], os autores discutem estratégias para auxiliar o ensinoaprendizagem nos cursos de Ciência da Computação e Engenharia de Software, da Universidade Federal do Pampa (UNIPAMPA), que possuem altos índices de repetência e evasão em componentes curriculares que envolvem o ensino de programação. Para tanto, os autores promoveram a criação espaços colaborativos de ensino e prática de programação, como o Coding Dojo.

Por outro lado, há esforços de mitigação do problema que consideram uma abordagem mais lúdica, semelhante a explorada neste trabalho. Em [Ribas et al. 2016] os autores discutem o impacto da formação prévia com o pensamento computacional, a lógica de programação e a matemática no desempenho em cursos superiores de informática. No caso, os autores relataram a experiência com alunos do curso de Ciência da Computação da UFRGS, com o ensino de linguagens de programação visual, a exemplo do Scratch. Em [Raiol et al. 2015] os autores relatam duas experiências com calouros de sistemas de informação da Universidade Federal Rural da Amazônia, utilizando o ambiente KTurtle/Logo, buscando contribuir para melhorar o desempenho em disciplina introdutória de programação. Em [Dantas et al. 2015] os autores apresentam o game chamado "Teddy Racer", que tem por objetivo auxiliar, de forma lúdica, o processo de ensino-aprendizagem em disciplinas relacionadas a lógica de programação, em cursos de informática e engenharias.

\subsection{Robótica Educacional}

A robótica educacional é o uso interligado dos recursos de software e hardware, como forma de estimular o aluno a planejar, projetar e criar soluções em diversos contextos educacionais, possibilitando a efetiva participação e a autonomia.

Nesse contexto, no trabalho de [de Oliveira Mattos et al. 2015], com o objetivo de despertar o interesse do público feminino para as áreas de tecnologia, os autores apresentam uma intervenção em uma escola pública com alunos do ensino médio. No caso, observou-se que a robótica é interessante como primeira ferramenta para o estudo de conceitos de programação e desperta o interesse para o público.

Em [Zilli and Lambert 2010] os autores apresentam a robótica para alunos do ensino médio e acadêmicos de engenharia elétrica, com dois objetivos: (i) atrair e incentivar os alunos do ensino médio para os cursos de graduação; e (ii) possibilitar aos acadêmicos uma estrutura de aula mais agradável e ao mesmo tempo testar uma proposta pedagógica onde os acadêmicos passam a ser os protagonistas na sala de aula. A proposta teve ótima 
aceitação, pois os alunos do ensino médio que antes não tinham interesse em ingressar na universidade, após as atividades $67 \%$ apresentaram interesse em uma graduação, enquanto que os acadêmicos se mostraram mais motivados e com o aprendizado mais significativo por meio da robótica.

\subsection{Computação Desplugada}

A Computação Desplugada é um método de ensino de ciência da computação, cujo objetivo é expor aos estudantes os conceitos computacionais e a forma como os cientistas da computação pensam, sem uso de computadores para isso [Bell et al. 2009]. As atividades propostas no método envolvem a resolução de diferentes problemas, onde os estudantes são expostos a conceitos fundamentais da ciência da computação.

Para o contexto da educação básica, há relatos de experiências do uso da Computação Desplugada no ensino médio e no ensino fundamental. Por exemplo, em [de Souza Barbosa et al. 2015] foi realizada uma "Gincana Desplugada" com alunos do primeiro ano do ensino médio, enquanto em [Raiol et al. 2016] os autores estudaram as diferenças da aplicação do material de Computação Desplugada em diferentes séries do ensino fundamental.

No ensino superior, há poucas aplicações da Computação Desplugada. Em [Gonçalves et al. 2013], inspirados no método da Computação Desplugada, os autores desenvolveram o jogo EvOU, usado para o ensino de lógica e conectores lógicos (conjunção, disjunção, negação e tabela verdade). Em [Bezerra 2014] a pesquisa relata a experiência de formação de uma turma do curso de Licenciatura em Computação, que aplicou e elaborou material de Computação Desplugada em uma escola de ensino fundamental.

\section{Materiais e Métodos}

A programação da Semana do Calouro 2018 ofertou seis oficinas de informática, com carga horária de seis horas de aula e 20 vagas cada, a citar: Computação Desplugada, Robótica Educacional, Algoritmos, Redes de Computadores, Programando com Python e Design Gráfico. Tanto os calouros, como os veteranos poderiam se inscrever nessas atividades, e todas as oficinas foram ministradas por veteranos. Neste trabalho, estamos interessados nas experiências das oficinas Robótica Educacional e Computação Desplugada. Nas Seções 3.1 e 3.2 são mostrados os planos de ensino das oficinas, enquanto que na Seção 3.3é discutido o método de avaliação utilizado.

\subsection{Oficina 1: Robótica Educacional}

O conteúdo de cada dia de aula foi estruturado em dois momentos, cada um dedicado ao ensino de um conceito novo. Para tanto, a oficina foi organizada com o seguinte conteúdo: aula exploratória do conjunto LEGO $^{\circledR}$ Mindstorms, conceitos de algoritmo, conceitos de lógica, noções de pensamento computacional, conceitos de entrada e saída de dados e conceitos de estruturas de repetição e seleção em um algoritmo. Os conteúdos foram organizados em quatro partes, sendo duas por dia. Cada aula recebeu um nome relativo ao conteúdo estudado, são elas: i) Hello World; ii) Compilando; iii) Você pode repetir? ; e iv) Fantoche.

Para que o trabalho se tornasse mais atrativo, despertando o interesse dos participantes, uma sequência didática e algumas estratégias foram adotadas. i) sequência 
didática: revisão da última aula com exercício; seguida de uma dinâmica lúdica do conteúdo da aula; testa-se o conhecimento em um exercícios prático e por fim, uma tarefa mais complexa para avaliação; ii) estratégias: trabalho em equipe com três membros; a gamificação, proposta para as equipes somarem pontos a cada desafio concluído;

Para o primeiro dia, em Hello World, é apresentado o conjunto LEGO ${ }^{\complement}$ Mindstorms e a estrutura da gamificação, elaborada no intuito de engajar as equipes, de proporcionar um ambiente de aprendizagem e competição saudável. O segundo momento da aula Compilando, propõe-se debater os conceitos de Entrada e Saída de Dados. A respeito dos termos Algoritmo, Lógica e Pensamento Computacional, os próprios não são explorados conceitualmente, mas podem ser associados diretamente aos exercícios práticos desenvolvidos pelos alunos durante as aulas, com a tentativa de gerar, por meio de debates, compreensão dos significados de forma colaborativa. Ainda como proposta de conteúdo do primeiro dia, os alunos exploram o conjunto LEGO $^{\circledR}$ Mindstorms e simularam o uso dos sensores e motores. Após esses momentos, as quatro equipes recebem o painel de pontuação para organização de suas conquistas durante as aulas, representadas por moedas, divididas em três cores, cada cor correspondendo um valor diferente, que tinham como principal critério de conquista o grau de complexidade de cada desafio proposto.

Para o segundo dia, realizou-se a criação de programações mais sofisticadas, com recursos de seleção e repetição de trechos do algoritmo. No primeiro momento da aula foi apresentado o conceito de Laço, Você pode repetir?, no qual foi possível desenvolver uma programação e repetir um código inteiro ou uma parte dele. No segundo momento da aula, Fantoche, foi elaborado soluções que estão ligadas ao processo de tomada de decisão.

Um dos destaques das aulas de programação com Robótica Educacional é a estrutura das aulas que compõem o método de ensino. A forma que foi pensada e elaborada visa a interação e participação ativa do aluno. Para [Libâneo 2017] há muitas classificações de métodos, cuja utilização depende dos objetivos-conteúdos-métodos das matérias, das peculiaridades do aluno e da criatividade do professor. Neste ínterim, houve a possibilidade de se trabalhar com mais de um método, e não somente de forma expositiva, alternando conforme a necessidade de cada momento da aula.

\subsection{Oficina 2: Computação Desplugada}

Esta oficina tinha como objetivo introduzir conceitos iniciais da ciência da computação utilizando atividades lúdicas, sem a utilização de computadores. Acredita-se que o contato com a Computação Desplugada é uma oportunidade para que os alunos possam obter um conhecimento prévio dos conteúdos que serão desenvolvidos durante a graduação. Para tanto, a oficina é baseada a no livro "Computer Science Unplugged: Ensinando Ciência da Computação Sem o Uso do Computador"'[Bell et al. 2011], que é dividido em três partes: i) Dados: A Matéria-prima - Representando a informação; ii) Colocando os Computadores para Trabalhar - Algoritmos; e iii) Dizendo aos Computadores o que fazer - Representando Procedimentos.

Para esta oficina foram selecionadas 6 atividades, que correspondem a três capítulos da primeira parte (Dados e Informação) e a três capítulos da segunda parte do livro (Algoritmos). As atividades selecionadas contemplam dois dos conceitos que mais 
são trabalhados nos semestres iniciais em ambos os cursos. A oficina foi realizada em dois dias consecutivos, cada dia com três horas de aula, esta por sua vez foi dividida na seguinte sequência didática: i) exposição de conceito; ii) atividade prática e iii) gincana.

No primeiro dia de oficina foram desenvolvidas as atividades: Contando os Pontos, que trabalha o conceito, utilização e conversão de números binários; Colorindo com Números que demonstra ao aluno como o computador representa e armazena imagens através de pixels e a atividade A Mágica de Virar as Cartas, que trata do método de detecção e correção de erros utilizado na troca e envio de dados.

Os exercícios e materiais utilizados estão disponíveis no livro para impressão, havendo pouca necessidade de produção de material de apoio. Em cada atividade, após expor um pequena introdução sobre o conteúdo a ser trabalhado, foi entregue ao aluno a folha de atividade. Em exemplo a isto, na atividade Contando os Pontos, o aluno deveria converter valores binários em valores decimais utilizando cartas, que auxiliam nesta conversão. O mesmo modelo foi utilizado nas demais atividades: inicialmente houve introdução ao conteúdo, seguida de atividade prática, e ao concluir a atividade, foi repassado ao aluno uma explicação final, relacionando a atividade realizada com o conceito computacional associado.

No segundo dia de oficina foram desenvolvidas as atividades: Batalha Naval, que demonstra alguns algoritmos de busca utilizados pelo computador; $O$ mais leve e o mais pesado, que aborda sobre algoritmos de ordenação, e a atividade Seja o mais rápido! que ilustra como o computador utiliza redes para ordenação de valores.

\subsection{Avaliação das Oficinas}

A avaliação das oficinas considerou três perspectivas: i) auto-avaliação do sentimento do aluno; ii) avaliação da oficina pelo aluno; e iii) avaliação da oficina pelos instrutores. Diariamente foram coletadas as fichas de auto-avaliação do sentimento de engajamento e motivação de cada aluno, como também o registro de observações da oficina pelos instrutores. No final da oficina (segundo dia), cada aluno respondeu de forma aberta um formulário de avaliação.

Para a avaliação do sentimento do aluno, ao final de cada uma das aulas, os alunos responderam uma autoavaliação não verbal chamada SAM (Self Assessment Manikin), para verificar o nível de aceitabilidade do trabalho a partir de três dimensões ligadas ao campo emocional: satisfação, motivação e controle. Para tal, o questionário se divide em três partes, representadas por três linhas com cinco figuras cada. A figura do centro representa uma resposta neutra, as duas primeiras figuras representam respostas positivas e as duas últimas, respostas negativas [Bradley and Lang 1994, de Macedo and Prietch 2013, de França et al. 2013]. A Figura 1(a) ilustra uma resposta obtida de um aluno no final da aula.

A avaliação da oficina pelo aluno foi realizada por meio de um formulário com três campos de resposta aberta. O primeiro campo o aluno deveria escrever de forma livre o que foi bom na oficina. No segundo campo, com espaço de resposta igual ao primeiro, o aluno deveria escrever de forma livre o que foi ruim na oficina. No terceiro campo, com o dobro do espaço de resposta dos dois primeiros campos, o aluno deveria escrever o que precisaria ser feito para melhorar uma próxima edição da oficina. A Figura 1(b) ilustra uma resposta obtida de um aluno no final do curso. 
VII Congresso Brasileiro de Informática na Educação (CBIE 2018)

Anais dos Workshops do VII Congresso Brasileiro de Informática na Educação (WCBIE 2018)

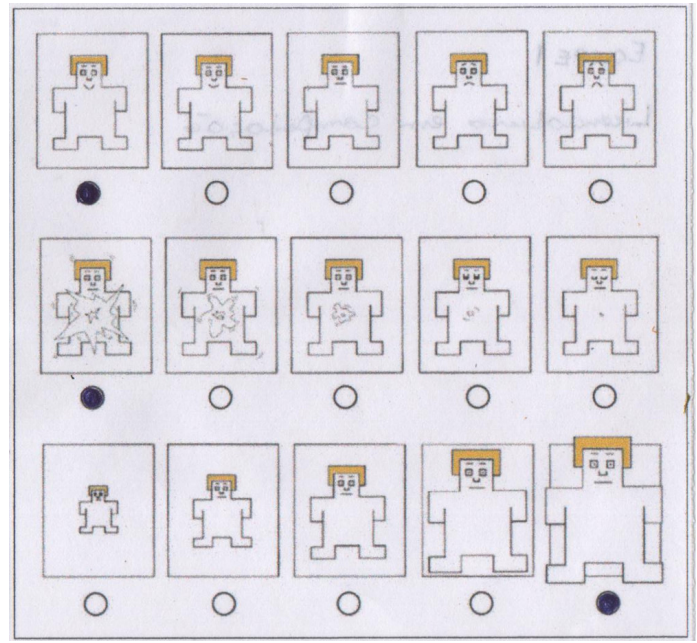

(a) Auto-avaliação do sentimento do aluno.

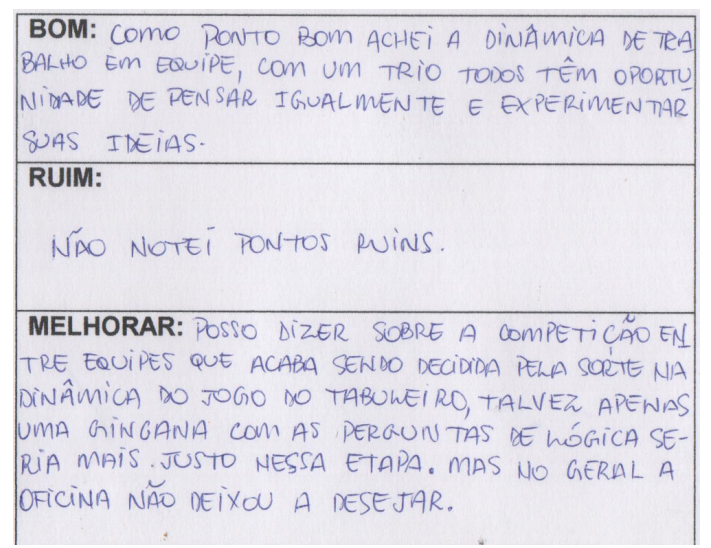

(b) Avaliação da oficina pelo aluno.

Figura 1. Avaliações do aluno

$\mathrm{Na}$ avaliação realizada pelos instrutores sobre as oficinas, três observações foram consideradas: i) a participação por meio do debate, que é quando o aluno questiona determinado conceito e expõe suas opiniões; ii) autonomia na execução das atividades, que se caracteriza quando o aluno realiza as tarefas e exercícios propostos; e iii) a intensidade do engajamento, "medida" pelo empenho na execução das tarefas.

\section{Resultados e Discussões}

Nesta seção, é apresentado os resultados organizados por oficina. Assim, na Seção 4.1 é resumido os resultados da oficina de Robótica Educacional, enquanto na Seção 4.2 são destacados os resultados da oficina de Computação Desplugada. Na Seção 4.3 é comentado como essas oficinas foram importantes para auxiliar na mitigação do problema da evasão e retenção de alunos de informática, por isso, é apresentado uma única discussão sobre os resultados.

\subsection{Oficina 1: Robótica Educacional}

\begin{tabular}{l|l|l|l||l|l|l||l|l|l}
\hline & \multicolumn{3}{|c||}{ Satisfação } & \multicolumn{3}{c||}{ Motivação } & \multicolumn{3}{c}{ Controle } \\
\hline & V- & VN & V+ & V- & VN & V+ & V- & VN & V+ \\
\hline Dia 1 & 0 & 0 & 13 & 0 & 0 & 13 & 0 & 4 & 9 \\
\hline Dia 2 & 0 & 0 & 13 & 0 & 0 & 13 & 0 & 3 & 10 \\
\hline Total & 0 & 0 & 26 & 0 & 0 & 26 & 0 & 7 & 19 \\
\hline
\end{tabular}

\section{Tabela 1. Resultado da auto-avaliação do aluno - Robótica Educacional}

A oficina contou com a participação de 15 alunos. Destes, o maior número era de calouros. Quanto a auto-avaliação do sentimento do aluno, as respostas foram coletadas e atribuídas a votos positivos ( $\mathrm{V}+$ ), negativos (V-) e votos neutros (VN), conforme detalhado na Tabela 1. No caso, a satisfação e motivação foram predominantemente positivas, enquanto que para o controle apenas $1 / 4$ se posicionaram de forma neutra. Vale ressaltar que no Dia 1, dois alunos se ausentaram antes da aplicação da avaliação, resultando 
VII Congresso Brasileiro de Informática na Educação (CBIE 2018)

Anais dos Workshops do VII Congresso Brasileiro de Informática na Educação (WCBIE 2018)

assim na obtenção de 13 respostas, e no Dia 2, dois alunos não compareceram à aula, ocasionando em 13 respostas registradas.

Quanto à avaliação do aluno obtida no final da oficina, os alunos opinaram sobre o que foi Bom, Ruim e o que poderia Melhorar. Analisando as 13 respostas, foi observado que a avaliação da oficina atingiu relevância, demonstrada pelos resultados positivos. Por exemplo, segue três respostas sobre cada uma das perguntas:

Bom "Muito Bom, a didática é realmente funciona, e nos faz ter o foco e o aprendizado necessário para lidar com os desafios propostos"

Ruim "Não observei pontos ruins"

Melhorar "Um ponto a melhorar seria a questão de mais eventos como estes na UFRA. (Oficinas, workshops, palestras, minicursos) voltados para T.I que possam incentivar, divulgar e despertar o interesse dos discentes."

Sobre as observações dos instrutores, foi destacado o trabalho em equipe, especialmente a forma de divisão das tarefas entre os membros dos grupos, onde todos puderam exercitar suas ideias durante as atividades. Foi notado que os alunos que participaram da oficina puderam compreender alguns conceitos pertinentes à sua área de formação, também fizeram observações gerais a respeito da aplicação dos conceitos de computação.

\subsection{Oficina 2: Computação Desplugada}

\begin{tabular}{l|l|l|l||l|l|l||l|l|l}
\hline & \multicolumn{3}{|c||}{ Satisfação } & \multicolumn{3}{c||}{ Motivação } & \multicolumn{3}{c}{ Controle } \\
\hline & V- & VN & V+ & V- & VN & V+ & V- & VN & V+ \\
\hline Dia 1 & 0 & 0 & 6 & 0 & 0 & 6 & 0 & 0 & 6 \\
\hline Dia 2 & 0 & 0 & 6 & 0 & 0 & 6 & 0 & 0 & 6 \\
\hline Total & 0 & 0 & 12 & 0 & 0 & 12 & 0 & 0 & 12 \\
\hline
\end{tabular}

Tabela 2. Resultado da auto-avaliação do aluno - Computação Desplugada

Para esta oficina o número de alunos foi abaixo de esperado, apenas seis vagas foram preenchidas, três por veteranos, e outros três por calouros. Quanto à autoavaliação do sentimento do aluno, as respostas foram coletadas e atribuídas a votos positivos (V+), negativos (V-) e votos neutros (VN), conforme detalhado na Tabela 2. No caso, a satisfação, motivação e controle foram predominantemente positivas.

Quanto a avaliação do aluno obtida no final da oficina, os alunos opinaram sobre o que foi Bom, Ruim e o que poderia Melhorar. Compilando as seis respostas, percebeuse que os participantes ficaram satisfeitos com as dinâmicas e atividades. Por exemplo, segue três respostas sobre cada uma das perguntas:

Bom "Todas as atividades realizadas foram de fácil compreensão e ajudará a entender o conteúdo programático"

Ruim "Pouco tempo para um vasto conteúdo"

Melhorar "Trabalhar em mais dias as atividades e colocar outras para fixar melhor"

Sobre as observações dos instrutores, foi destacado que os alunos foram participativos nas atividades aplicadas em sala, demonstrando que conseguiram compreender o que havia sido passado no momento da aula. Os alunos também manifestaram certa diversão com as atividades, ou seja, enquanto brincavam e competiam tentando resolver em menor tempo as atividades, também aprendiam. Outro destaque foi o trabalho em equipe, que é uma atitude estimulada durante a oficina para a conclusão das atividades, desafios e gincanas. 
VII Congresso Brasileiro de Informática na Educação (CBIE 2018)

Anais dos Workshops do VII Congresso Brasileiro de Informática na Educação (WCBIE 2018)

\subsection{Discussões}

Acredita-se que as duas oficinas discutidas neste trabalho devem ser ofertadas nas próximas edições da semana do calouro, por dois motivos: (i) são ricas em significado para os dois cursos de informática da Universidade (BSI e LC); (ii) e proporcionaram um aprendizado lúdico. Ambas desenvolvem no aluno conceitos de algoritmos, programação de computadores e o pensamento computacional, além de promoverem uma experiência de ensino que será o dia a dia do professor de informática nas escolas, nesse caso, vantagem específica para a clientela de Licenciatura em Computação.

Há uma diferença significativa no número de inscritos nas duas oficinas, que foi compreendida por ser em razão do grau de conhecimento dos inscritos com os conteúdos das duas oficinas, bem como da natural atração que o termo "robótica" promove na atualidade. Portanto, em uma próxima edição das oficinas, deseja-se uma divulgação com descrição mais detalhada, a fim de atrair mais inscrições. Por outro lado, apesar da evidente diferença no número de inscrições, notou-se o mesmo engajamento, visto que em ambas as oficinas os alunos mostraram-se motivados e satisfeitos.

Nas duas oficinas optou-se por utilizar o trabalho em equipe para obter o dinamismo dos alunos entre si e o conteúdo ministrado. Nota-se também que os conteúdos ministrados, associados as estratégias, podem incentivar os alunos a buscar cada vez mais informações sobre as diferentes áreas em que a computação é aplicada, bem como, entender a fundo o funcionamento de algorítimos e suas utilizações.

\section{Considerações Finais}

Este artigo relatou a experiência de duas oficinas na semana do Calouro 2018 da UFRA: Robótica Educacional e Computação Desplugada. Com seis horas/aula e objetivando ensinar conceitos iniciais da ciência da computação aos alunos de BSI e LC de forma lúdica, tais oficinas são um esforço da universidade em mitigar os problemas com a retenção e evasão de seu alunado.

A avaliação das oficinas considerou três perspectivas: i) a auto-avaliação do sentimento do aluno, ii) a avaliação das oficinas pelos alunos; e iii) a avaliação das oficinas pelos instrutores. Os resultados obtidos nas três perspectivas de avaliação com essa experiência são muito positivos, motivando para realização de ações semelhantes no futuro.

Como trabalhos futuros, esperamos avaliar os impactos dessa oficina na retenção dos alunos no primeiro ano de curso. Além disso, pretendemos comparar o desempenho nas disciplinas iniciais de programação de computadores entre os alunos inscritos na oficina, com os que não participaram das mesmas. Acreditamos que tais esforços possam corroborar com o planejamento de ações na UFRA e em outras IES.

\section{Referências}

Bell, T., Alexander, J., Freeman, I., and Grimley, M. (2009). Computer science unplugged: School students doing real computing without computers. The New Zealand Journal of Applied Computing and Information Technology, 13(1):20-29.

Bell, T., Witten, I. H., and Fellows, M. (2011). Computer science unplugged: Ensinando ciência da computação sem o uso do computador. Tradução coordenada por Luciano Porto Barreto. 
VII Congresso Brasileiro de Informática na Educação (CBIE 2018)

Anais dos Workshops do VII Congresso Brasileiro de Informática na Educação (WCBIE 2018)

Bezerra, F. (2014). Bem mais que os bits da computação desplugada. In Anais do Workshop de Informática na Escola, page 116.

Bradley, M. M. and Lang, P. J. (1994). Measuring emotion: the self-assessment manikin and the semantic differential. Journal of behavior therapy and experimental psychiatry, 25(1):49-59.

Dantas, D. O., Nobre, I. A. M., and Passos, M. L. S. (2015). Teddy racer: Lógica de programação e ludicidade. RENOTE, 13(2):10.

de França, R. S., da Silva, P. R. B., do Amaral, H. J. C., and de Lira, M. R. (2013). Avaliação de interfaces por usuários finais: Mensurando a qualidade afetiva e o impacto na aprendizagem de conteúdos. RENOTE, 11(1):10.

de Macedo, R. S. and Prietch, S. S. (2013). Utilizando a placa arduino como ferramenta de apoio ao ensino de programação. Revista de Exatas e TECnológicas, 4(2):51-56.

de Oliveira Mattos, G., da Silva, D. R. D., and Moreira, J. A. (2015). A utilização de kits de robótica como ferramenta para o ensino de programação à meninas do ensino médio. In XXIII Workshop sobre Educação em Informática, Recife, PE.

de Souza Barbosa, A. V., Neto, A. F. P., de Oliveira, R. N. R., da Costa, T. L. S., Souto, A. L., De Araújo, O., and Costa, F. V. S. (2015). O ensino de conceitos computacionais para alunos do ensino médio: relato de experiência de uma gincana e das estratégias utilizadas pelos alunos na resolução das atividades desplugadas. In XXI Workshop sobre Educação em Computação.

Franz, L. P., da Silva, J. P. S., and Cheiran, J. F. P. (2014). O uso de CODING DOJO no aprendizado colaborativo de programação de computadores. RENOTE, 12(2):9.

Gonçalves, D. A. S., da Silva, G. M., da Luz, R. S., and Silva, E. P. (2013). Relato de experiência de alunos do curso de licenciatura em computação do IFMG-Campus Ouro Branco na utilização de objetos de aprendizagem desplugados e do Scratch como instrumentos no ensino de programação. In Anais dos Workshops do Congresso Brasileiro de Informática na Educação.

Libâneo, J. (2017). Didática. Cortez Editora.

Raiol, A. A., Lima, A. C., Sousa, B., Sarges, J., Santos, R., and Bezerra, F. (2016). Experiências de ensino da computação desplugada em diferentes séries da educação fundamental maior. In XXIV WEI - Workshop sobre Educação em Computação, Porto Alegre, RS. SBC.

Raiol, A. A., Sarges, J., Souza, A., Silva, S., and BEZERRA, F. d. L. (2015). Resgatando a linguagem de programação Logo: Uma experiência com calouros no ensino superior. In XXIII Workshop sobre Educação em Informática, Brasilia, DF. SBC.

Ribas, E., Dal Bianco, G., and Lahm, R. A. (2016). Programação visual para introdução ao ensino de programação na educação superior: uma análise prática. RENOTE, 14:10.

Zilli, G. M. and Lambert, G. (2010). Desenvolvendo a educação através da robótica móvel: Uma proposta pedagógica para o ensino de engenharia. In XXXVIII Congresso Brasileiro de Educação em Engenharia, Fortaleza, CE. 